\title{
Effects of Nanocellulose on the Structure of Collagen: Insights from Molecular Dynamics Simulation and Umbrella Sampling
}

\section{Lulu Ning ( $\nabla$ ninglulu@sust.edu.cn )}

Shaanxi University of Science and Technology Xi\'an Campus: Shaanxi University of Science and Technology https://orcid.org/0000-0001-8608-3262

\section{Huaiqin Ma}

Shaanxi University of Science and Technology Xi\'an Campus: Shaanxi University of Science and Technology

\section{Qingwen Shi}

Shaanxi University of Science and Technology Xi\'an Campus: Shaanxi University of Science and Technology

\section{Xuhua Li}

Xian Jiaotong University: Xi'an Jiaotong University

Junli Ren

Shaanxi University of Science and Technology Xi\'an Campus: Shaanxi University of Science and Technology

\section{Zhijian Li}

Shaanxi University of Science and Technology Xi\'an Campus: Shaanxi University of Science and Technology

\section{Research Article}

Keywords: nanocellulose, collagen, molecular dynamics simulation, umbrella sampling

Posted Date: January 24th, 2022

DOI: https://doi.org/10.21203/rs.3.rs-1242782/v1

License: (c) (i) This work is licensed under a Creative Commons Attribution 4.0 International License. Read Full License 


\title{
Effects of Nanocellulose on the Structure of Collagen: Insights from Molecular Dynamics
}

\section{Simulation and Umbrella Sampling}

\author{
Huaiqin $\mathrm{Ma}^{1}$, Qingwen $\mathrm{Shi}^{1}$, Xuhua $\mathrm{Li}^{2}$, Junli Ren ${ }^{3}$, Zhijian $\mathrm{Li}^{1 *}$, Lulu Ning ${ }^{1 *}$ \\ ${ }^{1}$ College of Bioresources Chemical and Materials Engineering, Shaanxi University of Science \& \\ Technology, Xi'an 710021, China
}

${ }^{2}$ MOE Key Laboratory for Nonequilibrium Synthesis and Modulation of Condensed Matter, School of Physics, Xi'an Jiaotong University, Xi'an 710049, China

${ }^{3}$ Information Center, Shaanxi University of Science \& Technology, Xi'an 710021, China

"Corresponding author’s e-mail address: zjli@sust.edu.cn, ninglulu@sust.edu.cn

ABSTRACT: Collagen-nanocellulose composites have been widely used in biomedicine and tissue engineering. However, the detailed mechanism underlying the effects of nanocellulose on the structure of collagen hasn't been elucidated. As the main component of skin tissue, the conformational disturbance of collagen triggered by nanocellulose may shed light on the biocompatibility of nanocellulose. Therefore, molecular dynamics simulations were carried out to gain insights into the interactions between nanocellulose and collagen. Four different crystal planes of cellulose ((110), (100), (1-10), (010)) have been constructed and the adsorption of collagen onto the four faces has been investigated respectively. It has been found that the structure of collagen remained intact during the binding without chain separation. The intactness of collagen supported the point that the nanocellulose has good biocompatibility. The results derived from umbrella sampling showed that (110) and (1-10) faces exhibit the strongest affinity with collagen, which may be attributed to its hydrophilicity and rather flat surfaces. The hydrophobicity of (100) facet and roughness of (010) facet diminished the affinity with collagen. The occupancy of hydrogen bonds was low and hydrogen bonding interactions fail to make significant contributions to the binding of nanocellulose and collagen. These findings provided insights into the interactions between cellulose and collagen at an atomic level, which may guide the design and fabrication of collagennanocellulose composites. Furthermore, the biocompatibility of nanocellulose validated in the study may help promote the biological application of nanocellulose involved composites.

Keywords: nanocellulose, collagen, molecular dynamics simulation, umbrella sampling 
As a valuable new type of green biological nanomaterial, nanocellulose has the advantages of a fine nanostructure, good mechanical strength, and low thermal expansion coefficient, as well as recoverability and sustainability (Fernandes and Madhuranthakam 2020; Zhang et al. 2019). Moreover, nanocellulose in the form of nanoparticles, tablets, gas-liquid gel system, and fiber membrane system is widely used in the field of energy storage (Chen et al. 2018), wastewater treatment (Tang et al. 2019), 3D bioprinting(Dorishetty et al. 2020), biosensor(Golmohammadi et al. 2017), drug delivery(Salimi et al. 2019) and other biological fields. Compared with graphene (Luan, Huynh and Zhou 2016), graphene oxide (He et al. 2019), molybdenum disulfide (Gu et al. 2016; Gu et al. 2017), carbon nanotube(El-Sayed et al. 2016), and other 2D nanomaterials, nanocellulose as a carrier material has a lower nanotoxicity, which makes it more widely available. Although nanocellulose and its derivatives have been widely concerned in medicine and biological tissue, the long-term retention of nanocellulose in the human body makes its toxicity study very important due to the lack of cellulose-degrading enzymes in the human body.

Collagen can also be a candidate for biomaterials such as tissue-engineered scaffolds and wound dressings (Lee et al. 2019; Ge et al. 2018; Sorushanova et al. 2019). However, the application of pure collagen materials is limited due to their low water resistance, fast biodegradation perishability, and poor thermal stability (Ge et al. 2018). While cellulose and collagen nanocomposite materials overcome the weaknesses of pure collagen materials. Cellulose and its derivatives can be widely used to strengthen various polymer matrix materials due to their high specific surface area, high crystallinity, low density, and high elastic modulus (Manhas et al. 2015; Salimi et al. 2019; Liu et al. 2018; Li et al. 2017). Researchers have proved that collagen/nanocellulose composite has good properties and stability better than pure collagen. Animal experimental studies (Liu et al. 2020b; Liu et al. 2020a) (Collagen/cellulose nanofiber hydrogel scaffold: physical, mechanical and cell biocompatibility properties; A 3D porous microsphere with multistage structure and component based on bacterial cellulose and collagen for bone tissue engineering (Zhang et al. 2020); demonstrated that collagen and nanocellulose composite is a promising material for wound dressings and tissue engineering scaffolds. 
composites have a broader application prospect. However, the interactions between nanocellulose and collagen, which are significantly related to the strength of composites, are still obscure. Furthermore, in vivo and in vitro experiments have shown that nanocellulose and its derivatives have adverse effects on intestinal microorganisms (DeLoid et al. 2019), liver cells (Otuechere et al. 2020), and lung cells(Sai and Fujita 2020). In addition, nanocellulose biological dressings and tissue-engineered materials, as the main components of human tissues, will directly interact with collagen when they contact the human body. Therefore, it is necessary to study the toxicity of nanocellulose.

In this study, molecular dynamics simulations were carried out to study the interactions between collagen and nanocellulose. Nanocellulose materials are char0acterized by a high degree of crystallinity thus it is feasible to employ cellulose crystal to model the properties of nanocellulose. Native crystalline cellulose contains a mixture of faces, which complicates the direct assignment of cellulose-collagen interactions to a specific face by experimental methods such as NMR spectroscopy. Molecular dynamics simulations provide a good solution to bypass this limitation. A single crystal face in nanoscale can be constructed by molecular modeling. Molecular dynamics simulations have been carried out by Crowley et al. to study the interactions between cellulose and lignin and gain insights into quantitative relationships between different cellulose faces and specific lignin chemistries (Vermaas, Crowley and Beckham 2019). Moreover, molecular dynamics simulations have also been used to probe the interactions between water molecules and cellulose, which shed light on the wetting mechanisms of cellulose(Malaspina and Faraudo 2019); Cellulose nanocrystals produced using recyclable sulfuric acid as hydrolysis media and their wetting molecular dynamics simulation; Molecular simulation of surface reorganization and wetting in crystalline cellulose I and II, Wetting the (110) and (100) Surfaces of I $\beta$ Cellulose Studied by Molecular Dynamics. Furthermore, many the interaction between two-dimensional nanomaterials and biomacromolecules have also been investigated by molecular dynamics simulations. Zhou et al., adopted molecular dynamics simulation (MD) to conduct thorough research on the interfacial properties of two-dimensional nanomaterials and found that graphene (Luan et al. 2016), graphene oxide(He et al. 2019; Mathesh et al. 2016), defective graphene(Gu et al. 2019) and other carbon nanomaterials can induce protein and nucleic acid denaturation, which provided theoretical support for the potential nanotoxicity of nanomaterials when used in biological systems. , 
model nanocellulose. Collagen type I was employed as the model collagen, which is the most abundant and widely distributed natural structural protein in the human body (Lin and Liu 2006). The adsorption of collagen on different crystal planes of cellulose was simulated by molecular dynamics. Then the structural changes and specific interactions were characterized in detail. This study investigated the interactions between nanocellulose and collagen at a molecular scale and evaluated the structural changes of collagen, which revealed the possible biological effects of nanocellulose and provided theoretical guidance for the design of nanocellulose-collagen complex at the same time.

\section{Methods}

The initial structure of collagen is obtained by extracting three chains from the crystal structure (Berisio et al. 2009) (PDB code 1K6F) as shown in Fig. 1(B) All nanocellulose crystal faces of different sizes (Fig. 1A) were constructed using Cellulose-Builder (Gomes and Skaf 2012). The thickness of (100), (110), (1-10) and (010) varied to weaken the effects of surface tension. Collagen was placed above the surface of the nanocellulose crystal at different angles $\left(0^{\circ}, 30^{\circ}\right.$ and $\left.45^{\circ}\right)$ in VMD software(Humphrey, Dalke and Schulten 1996) to form the initial coordinates of the simulation system and the minimum distances between cellulose faces and collagens were ranging from $0.8 \mathrm{~nm}$ to $1 \mathrm{~nm}$ (Fig. 2A). As shown in Fig. 2(A), three parallel simulations of each system were carried out for $500 \mathrm{~ns}$. The composite system was solvated in a cubic box with a TIP3P water model (Mark and Nilsson 2001; Jorgensen et al. 1983) and modeled by a CHARMM36 force field (Lee et al. 2014; Boonstra, Onck and van der Giessen 2016).

(A)

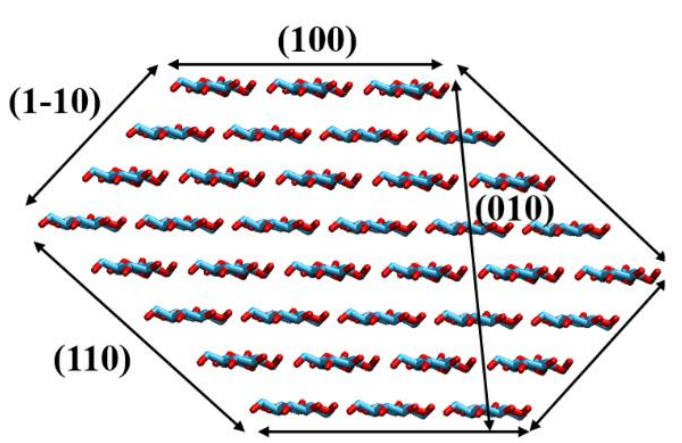

(B)

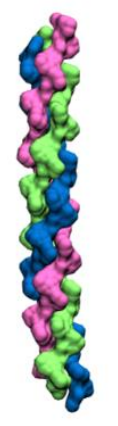

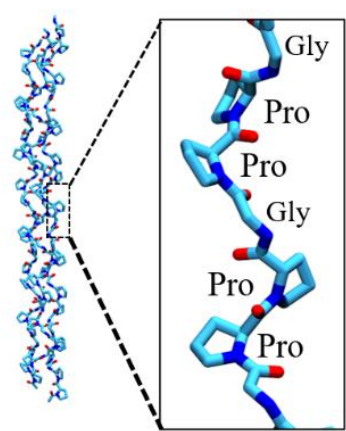

Fig. 1. (A) The crystalline plane of cellulose I $\beta$. (B) Collagen in initial configuration (PDB ID: 1K6F).

All MD simulations were performed in GROMACS-5.1(Berendsen, Spoel and Drunen 1995; Van Der Spoel et al. 2005) package All the systems were equilibrated carefully in the beginning of simulation. The energy minimization process was carried out with 1000 cycles of steepest descent and 1,000 cycles 

ensemble and $5 \mathrm{~ns}$ in the NPT ensemble with the heavy atoms of protein and cellulose fixed. Finally, 500 ns production runs were simulated in the NPT ensemble with the restriction of the protein released. The long-range electrostatic interactions were treated by the particle mesh Ewald (PME) method (Petersen 1995), while the short-range van der Waals interactions were calculated with a cutoff distance of $1.0 \mathrm{~nm}$. All covalent bonds containing hydrogen atoms were constrained by the LINCS algorithm (Hess et al. 2008). The V-rescale thermostatic (Berendsen et al. 1984) was used to heat the system to $300 \mathrm{~K}$ and the Parrinello-Rahman Pressure coupling (Parrinello and Rahman 1981; Nosé and Klein 2006) kept the system pressure at 1 bar. The integration step size of the simulation process is $2 \mathrm{fs}$. Periodic boundary conditions were applied in all directions with glycosidic bonds formed between mirror images.

The potential of mean force (Roux 1995) (PMF) obtained by pulling simulation and umbrella sampling (Hub 2015) was used to calculate the binding free energy of the system. The cellulose surface was used as a reference point and a harmonic potential was applied to the collagen as a pulling point. The last frame of the MD simulations was selected as the initial conformation, 300 ps umbrella traction was provided for collagen along the z-axis to increase the center of mass (COM) distance between collagen and cellulose. The spring constant used was $2000 \mathrm{~kJ} \mathrm{~mol}^{-1} \mathrm{~nm}^{2}$ and the pull rate was $0.01 \mathrm{~nm} / \mathrm{ps}$. More than 13 umbrella sampling windows were selected according to the interval size of COM values. 1ns of simulations in NPT was performed on each sample, then $10 \mathrm{~ns}$ of MD process was carried out. Finally, weighted histogram analysis (Hub 2015) (WHAM) was used to calculate PMF.

The relevant modules in GROMACS were used to calculate the backbone root mean square deviation (RMSD) and backbone root mean square fluctuation (RMSF) of proteins during the whole simulation process. G_hbond was used to calculate the number of hydrogen bonds the distance of 0.30 nm and angle $30^{\circ}$ as criterial.

\section{Results and discussions}

\subsection{Structural of collagen remained intact on nanocellulose}

In the initial simulation system, the distance between protein and cellulose crystal faces was controlled between $0.8-0.1 \mathrm{~nm}$ as shown in Fig. $2 \mathrm{~A}$ and three groups of different model analyses were conducted for each crystal face. Although the hydrophilicity and hydrophobicity of the selected cellulose 

simulation.

\section{(A)}

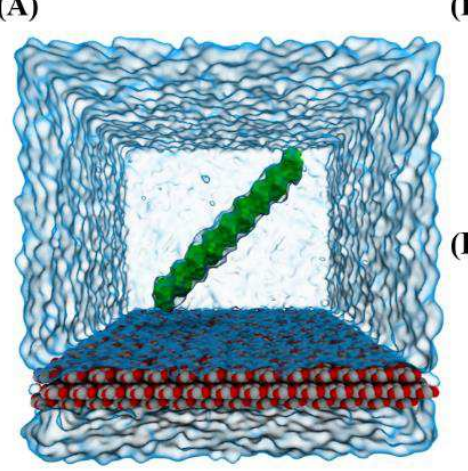

(B)

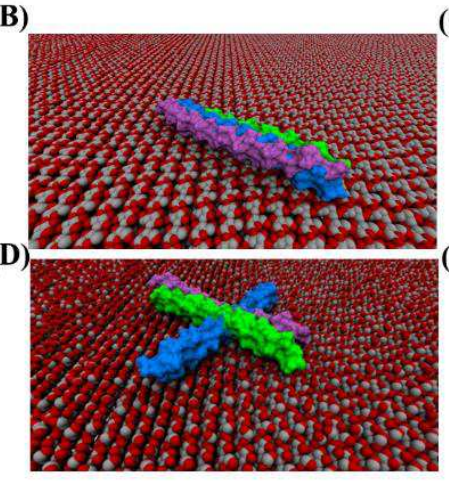

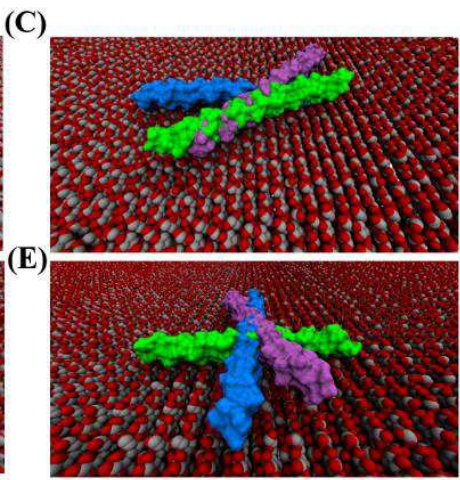

140

Fig. 2. (A) The initial system configuration of (100) planes. The water box is rendered with a cyan surface. (B-E) Snapshots of four crystal planes at 500ns. The protein shows green $\left(0^{\circ}\right)$, purple $\left(30^{\circ}\right)$, and blue $\left(45^{\circ}\right)$ to indicate three tracks, and the cellulose crystal plane is shown in sphere (carbon, gray; oxygen, red), hydrogen is not shown for clarity.

The calculation of the root-mean-square deviation of the protein was carried out to quantitatively measure the change of collagen structure during the simulation. RMSD values of collagen on different nanocellulose crystal faces are kept between $0.2 \mathrm{~nm}-0.3 \mathrm{~nm}$ as shown in Fig. 3(A-D). The profiles of RMSD exhibit very small fluctuation during the whole process. RMSD values in this range indicated that the protein structure did not change significantly during the adsorption process. Furthermore, RMSF was calculated to evaluate the free movement degree of each residue in collagen molecules. As shown in Fig. 3(E-H), the profiles of RMSF has good accordance with each other regardless of the properties of different cellulose faces. Each chain of the collagen model is composed of 29 residues and the peak of the line represents the end of each chain, which indicates that the two ends of the polypeptide chain are more flexible and the structure of other residues located in the middle of collagen is stable. In conclusion, the overall structure of collagen was not damaged during the process of adsorption on different crystal faces of cellulose. It is widely recognized that the change of protein structure induces the loss of its biological function (Feng et al. 2017), thus it can be inferred that nanocellulose has good biocompatibility. 


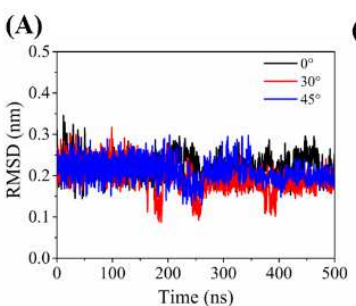

(E)

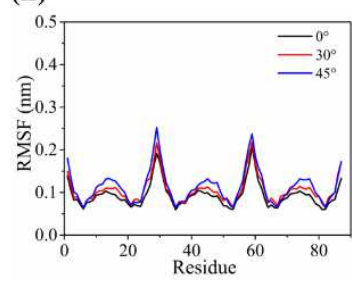

(B)

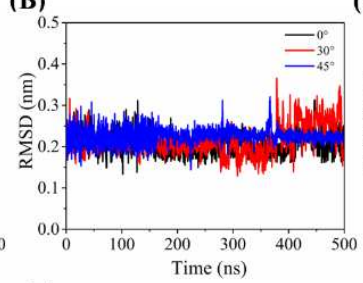

(F)

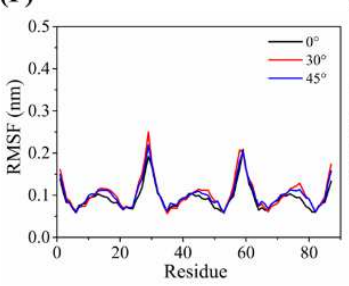

(C)

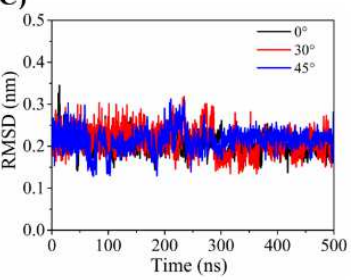

(G)

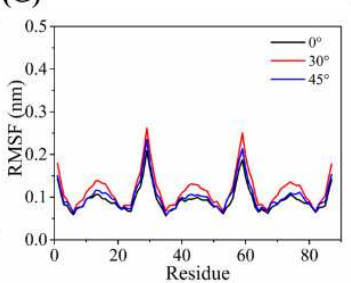

(D)

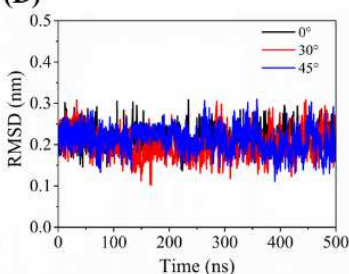

(H)

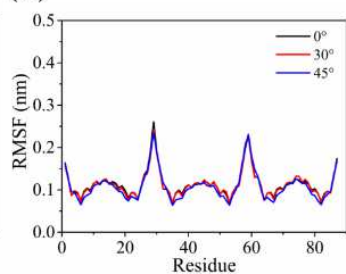

Fig. 3. (A-D) Time evolution of root mean squared deviation (RMSD) values of collagen on (100), (110), (1-10) and (010) faces over time, respectively. (E-H) root mean square fluctuation (RMSF) variation of each residue that constitutes collagen on different crystal planes.

\subsection{Evolution of the secondary structure of collagen}

The structure of proteins plays an important role in their activity and biological effects. To analyze the structural changes of collagen in the process of adsorption in more detail, the Ramachandran plot (Hooft, Sander and Vriend 1997) was used to characterize the changes in the secondary structure of proteins. Collagen is a coil, but one with distinct tertiary and quaternary structures: three separate polypeptides, called $\alpha$ chains are supertwisted about each other. The superhelical twisting is right-handed in collagen, opposite in sense to the left-handed helix of the $\alpha$ chains. Thus, it is ambiguous to evaluate the secondary structure of collagen by designating the structure helix, sheet, or coil. Therefore, Ramachandran plots were employed. The collagen helix is a unique secondary structure with $\mathrm{Phi}=-$ $51^{\circ}$ and Psi $=+153^{\circ}$, which is quite distinct from the $\alpha$ helix (Köppen, Ohler and Langel 2007). As shown in Fig. 4, Ramachandran plots of the last frames extracted from MD simulations display similar characteristics and most of Phi and Psi values are confined within the region corresponding to the structure of collagen. Random coil structures have also been observed with Phi and Psi values appearing around $(-90,-180)$. it was found by comparison that the Phi and Psi angles of collagen not significantly deviated (Fig. 4A-D) from the specified collagen conformation values during the adsorption of collagen in different crystal planes of cellulose. Therefore, it is indicated that all models exhibit partial structural interruption but are not damaged during adsorption. 
(A)

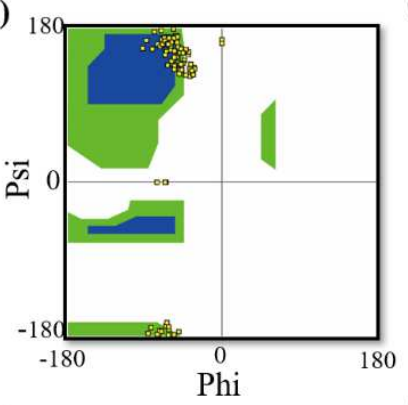

(C)

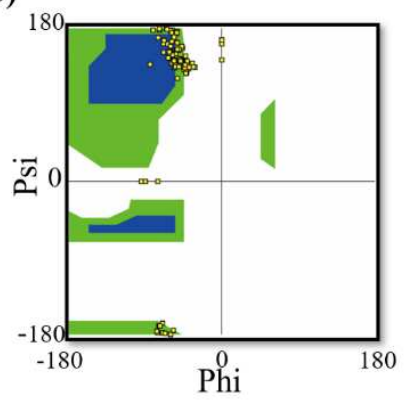

(B)

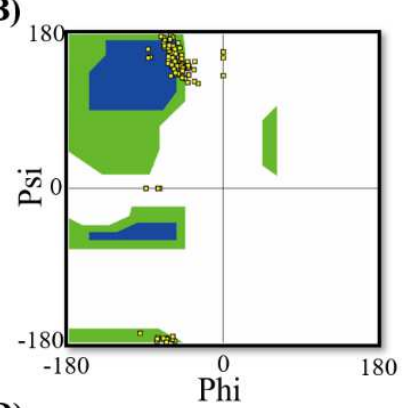

(D)

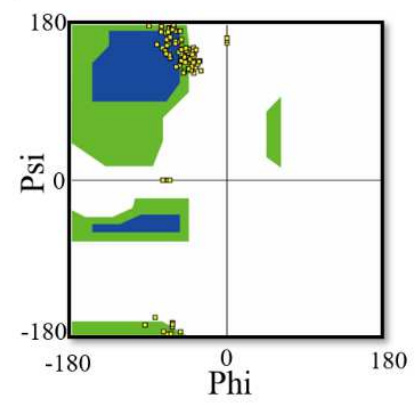

Fig. 4. The Ramachandran plots of collagens adsorbed on the planes (100) (A), (110) (B), (1-10) (C), and (010) (D).

To further investigate the effects of nanocellulose on the global structure of collagen, principal component analysis contour maps were constructed. which is also an effective method to analyze protein structure. Fig. 5 displays the free energy contour map of collagen after PCA in 12 simulated trajectories. As shown in Fig5, the free energy contour values of each model are located in a similar region with only one global minimum, which indicates that the effects of different crystal faces on collagen structures are too little to induce obvious changes. All the global minima of the $12 \mathrm{MD}$ simulations are restricted within narrow ranges with RMSD about $0.23 \mathrm{~nm}$ and $\mathrm{Rg}$ about $0.42 \mathrm{~nm}$, which further indicates the intactness of collagen on the surface of nanocellulose. The conformational space of collagen on the surface of nanocellulose provided information about the structural state of collagen in nanocellulose-collagen composites. Moreover, the study also partially evaluated the biological effects of nanocellulose when it is applied in the human body as a biomedical material. It exerted limited influence on the structure of collagen, which further validated the biocompatibility of nanocellulose. 
(A)

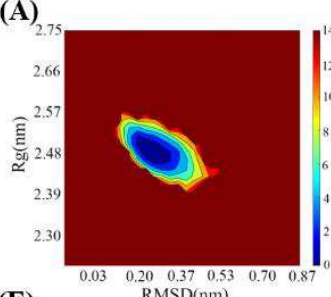

(E)

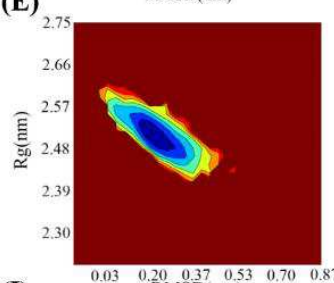

(I)

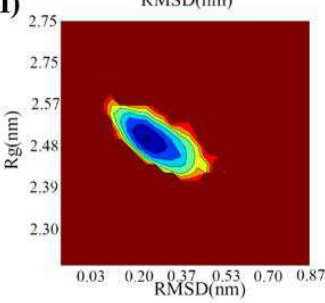

(B)

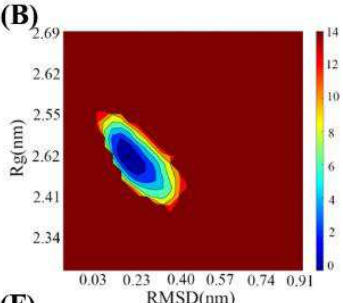

(F)

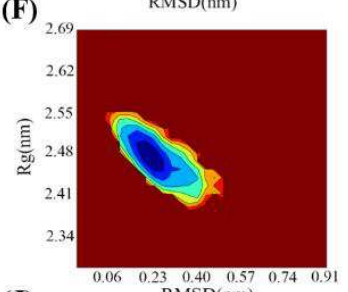

(J)

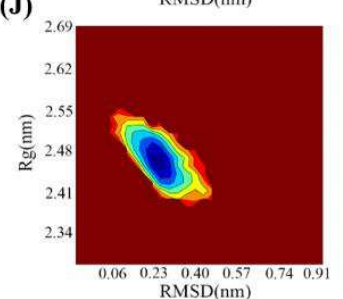

(C)

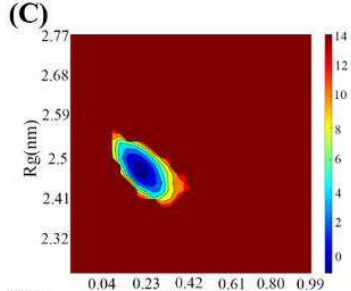

(G)

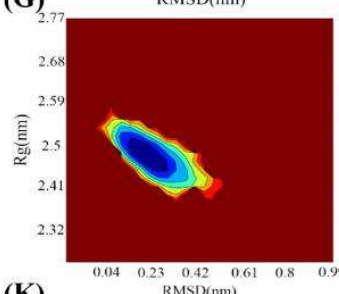

(K)

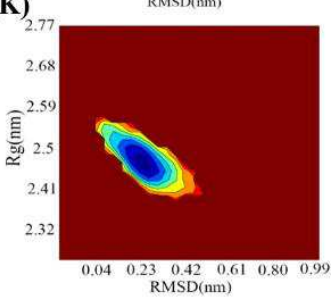

(D)

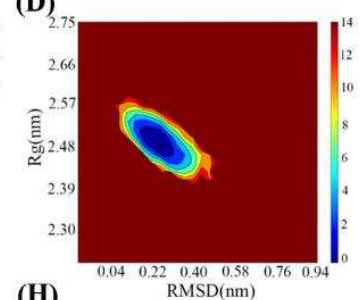

(H)

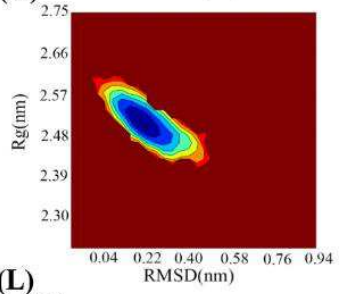

(L)

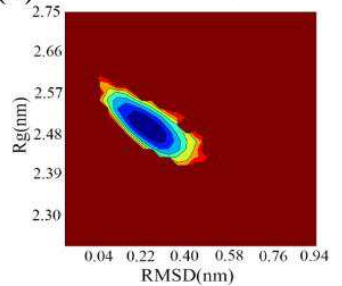

195

Fig. 5. Free energy landscape obtained from principal PCA of all models. From left to right, (100), (110), (1-10), and (010) sides. The horizontal rows from left to right are (100), (110), (1-10), and (010), respectively. The vertical rows from top to bottom represent $0^{\circ}, 30^{\circ}$, and $45^{\circ}$.

\subsection{Interaction between collagen and nanocelluloses during adsorption}

To understand the adsorption of collagen onto nanocellulose, umbrella sampling simulations were performed for all the four faces. Prior to the umbrella sampling simulation, the centers of all the cellulose models move to original points. Reaction coordinates were corrected by deducting half of its thickness in order to eliminate the effects of different thicknesses of cellulose slab and better illuminate the properties of different surface morphologies. As shown in Fig. 6(A), the lowest PMF values are found near the layer surface for all the systems, indicating that collagen tends to bind to cellulose. PMF shows that free energies are close for faces (110) and (1-10) with values of $-15.5 \mathrm{kcal} / \mathrm{mol}$ and $-14.8 \mathrm{kcal} / \mathrm{mol}$ respectively, which imply that almost the same adsorption strength of collagen onto the (110) and (1-10) surface. Free energies of (100) $(-9.5 \mathrm{kcal} / \mathrm{mol})$ are a little higher than those of (110) and (1-10) faces, indicating that the affinity between (100) layer and collagen are slightly weaker. In contrast with PMF profiles of (110), (1-10), and (100), the binding energies of (010) are much lower $(-5.2 \mathrm{kcal} / \mathrm{mol})$. These free energies derived from umbrella samplings indicate that collagen tends to migrate toward all the faces 
of cellulose. In particular, (110) and (1-10) faces displaying the strongest affinity to collagen. (100) face,

which is more hydrophobic, displays weaker adsorptive capability with collagen. Due to the structural anisotropy of cellulose, $I \beta(110)$ and $I \beta(1-10)$ faces are hydrophilic while $I \beta(100)$ face is hydrophobic.

The chain of collagen is composed of repeating tripeptide sequence Gly-Pro-Pro, which are all polar amino acids. Polar interactions between hydrophilic cellulose faces and collagen enhance their affinity. Though (010) face is hydrophilic, the interaction between these faces and collagen is the weakest among the four systems, which seems quite counterintuitive. The origin of this behavior is attributed to the topography of (010) faces, which hinders the binding of collagen with half of the hydroxyl grouping shielded. The steric hindrance weakens the interactions between collagen and (010) face, which is consistent with the results of the analysis of contact number. (110) and (1-10) are more hydrophilic than (100) but less rough than (010) face (Fig. 7E-H), which may result in a stronger affinity with collagen.
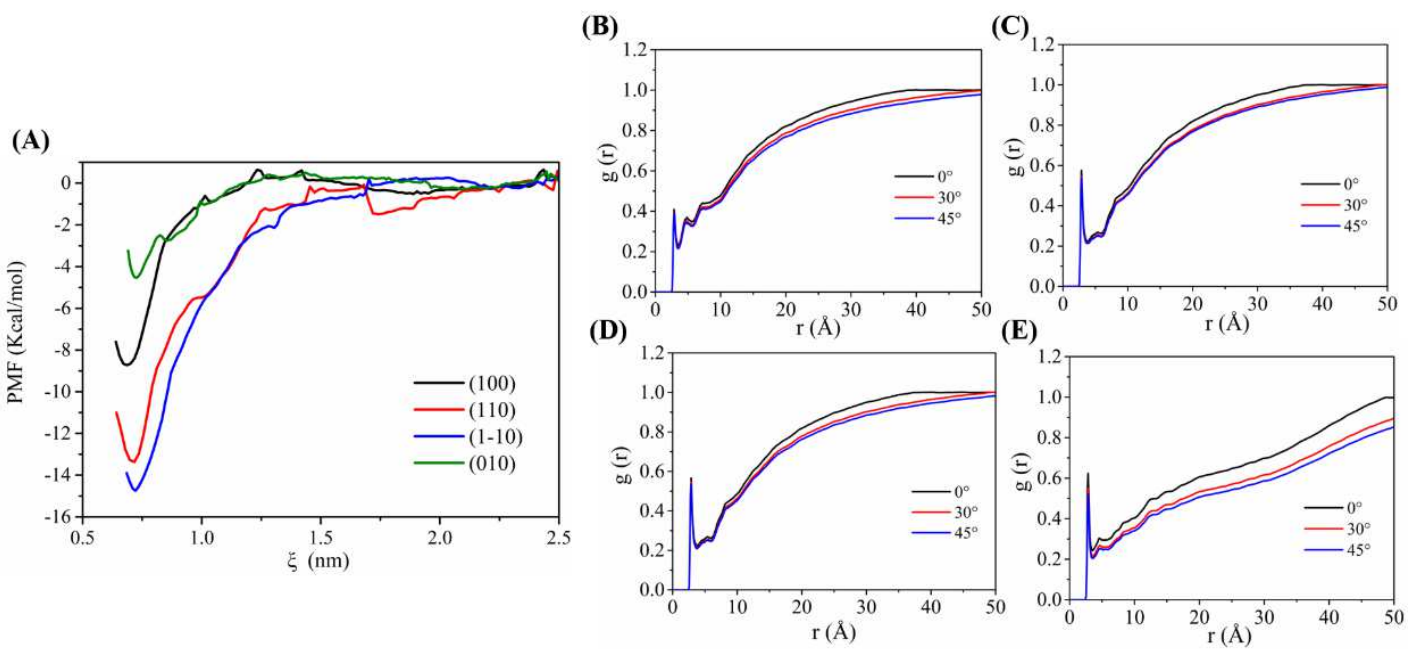

Fig. 6. (A) Potential of mean force plots describing the binding of collagen and cellulose. The radial distribution function of oxygen in exposed hydroxyl groups on water and cellulose surfaces, with cellulose surfaces represented by (B) (100), (C) (110), (D) (1-10) and (E) (010) respectively.

To further validate the deduction, radial distributions of water molecules were calculated to evaluate the hydrophilicity of the four faces. As shown in Fig. 6(B-E), their first peak positions occur at the same position $3.8 \AA$, respectively. The height of the first g (r) peak belonging to (100) face is about 0.4 , which is significantly lower than that of (110), (1-10) and (010) faces with the heights of the first peak all about

231 0.6. Thus, the heights of the first peaks describe a distinguishable difference in hydrophilicity among the four faces and the surface hydration of (100) face is weaker than the other three faces. To evaluate the effects of surface morphology on the interaction between collagen and cellulose, the contact number of 
heavy atoms was calculated with $0.5 \mathrm{~nm}$ as the threshold. In general, the loading of collagen on the cellulose surface was fast with molecules of collagen adsorbed on cellulose within $200 \mathrm{~ns}$. Based on the heavy atom contact numbers between collagen and cellulose, it has been deduced that there is an obvious correlation between surface roughness and contact numbers. As shown in Figure 7, (100) face displays the largest contact number with the smoothest surface, while, (010) face exhibits the least contact number with the greatest surface roughness. (110) and (1-10) faces are in between with the contact numbers larger than that of (100) face but less than that of (010) face. As the fundamental part of molecular interactions, the block of direct contact between collagen and (010) face impaired their affinity. The combinations of mediocre hydrophilicity and smoothness made (110) and (1-10) faces stand out from the other two crystal facets in the adsorption process.

(A)

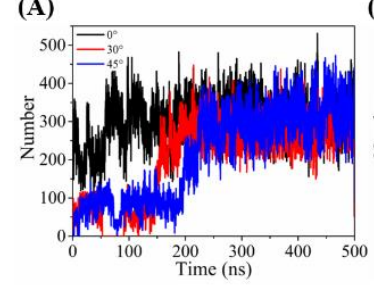

(E)
(B)

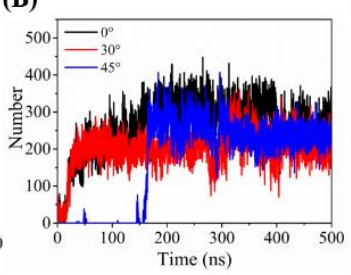

(F)
(C)

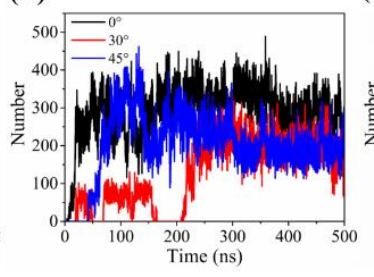

(G)

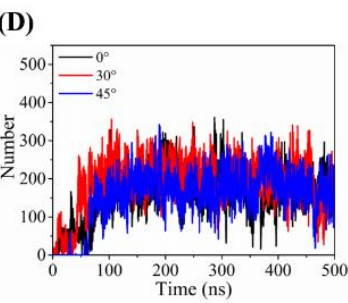

(H)

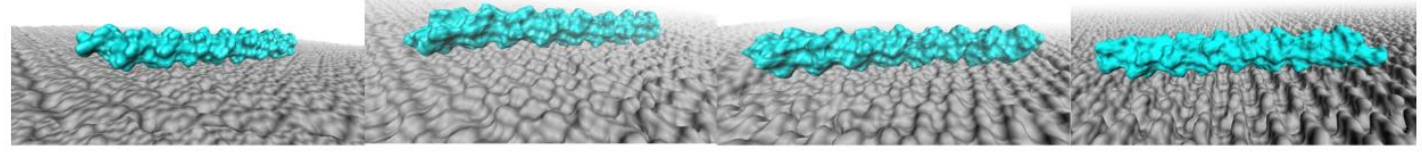

Fig. 7. Change of heavy atomic contact number of collagen and (100) (A), (110) (B), (1-10) (C), (010)

246 (B) faces over time. (E-H) The last frame trajectory of the model when the collagen was $0^{\circ}$ on the four crystal planes. Collagen is blue, cellulose overall structure is gray.

Cellulose molecules contain a large number of free hydroxyl groups, which might be involved in hydrogen bonding interactions. Therefore, the average occupancy of hydrogen bonds between the collagen and cellulose in the four systems was calculated respectively. As shown in Fig. 8, the values of hydrogen bonding occupancies are all lower than $12 \%$, which indicates that hydrogen bonding interaction is not the predominant force driving the binding of collagen and cellulose. (010) face of cellulose exhibits the highest tendency to form hydrogen bonds with collagen, which is consistent with the outward orientation of its surface hydroxyl groups. The hydrophobic (100) facet is less inclined to be involved in hydrogen bonding interactions with hydroxyl groups mainly forming interchain hydrogen bonds. (110) and (1-10) facets have a median performance in hydrogen bonding interactions. Collagen adsorption may 


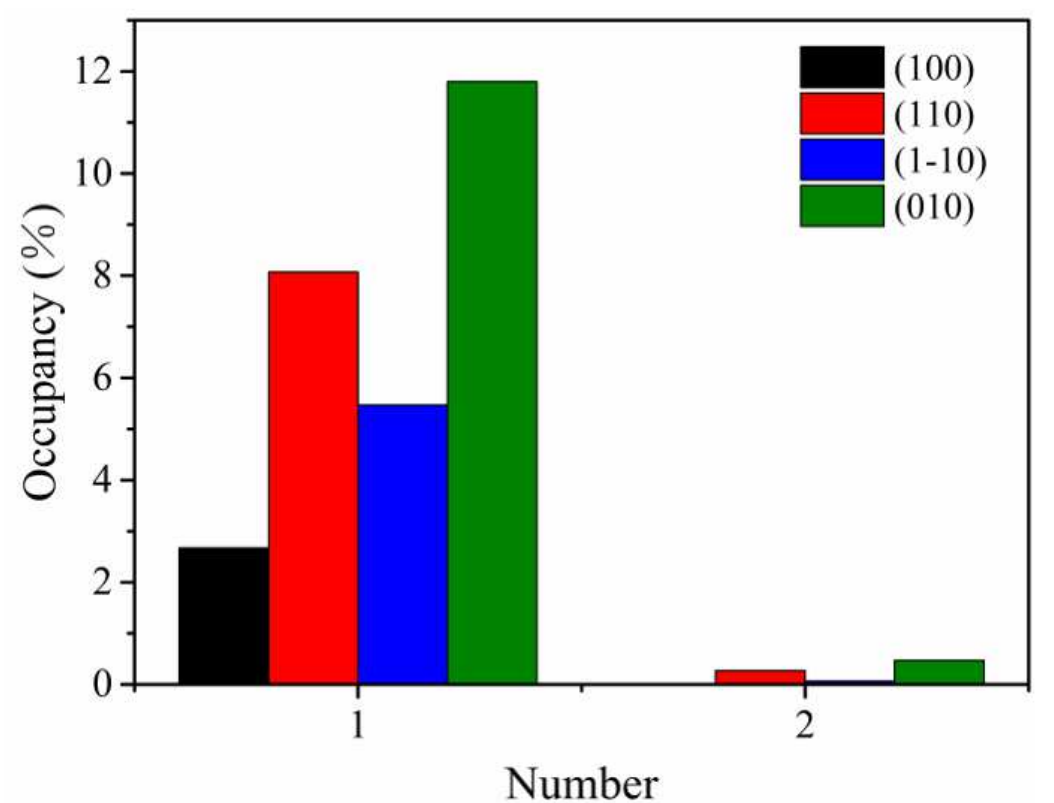

Fig. 8. Hydrogen bond occupancy between collagen and cellulose crystalline faces.

\section{Conclusion}

In this study, molecular dynamics simulations were carried out to investigate the adsorption behavior of collagen on the ideal nanocelluloses surface. It has been observed that the structural integrity of collagen has been maintained in the process of adsorption, which may shed light on the biocompatibility of cellulose. (110) and (1-10) crystal faces exhibited the strongest affinity with collagen, which was attributed to the combination of hydrophilicity and roughness. Hydrogen bonding interactions are not frequent and are not the predominant force driving the binding of collagen and nanocellulose. This study provides theoretical guidance for the design and fabrication of collagen-nanocellulose composites. Furthermore, the intactness of collagen structure supported the viewpoint that nanocellulose is quite biocompatible.

\section{Acknowledgments}

The authors acknowledge the financial supports from the Natural Science Basic Research Plan in Shaanxi

Province of China (2021JQ-537). 


\section{References}

Berendsen, H. J. C., J. P. M. Postma, W. F. van Gunsteren, A. DiNola \& J. R. Haak (1984) Molecular dynamics with coupling to an external bath. The Journal of Chemical Physics 81:3684-3690. http://doi.org/10.1063/1.448118

Berendsen, H. J. C., D. v. d. Spoel \& R. v. Drunen (1995) GROMACS: A message-passing parallel molecular dynamics implementation. Computer Physics Communications

Berisio, R., L. Vitagliano, L. Mazzarella \& A. Zagari (2009) Crystal structure of the collagen triple helix model [(Pro-Pro-Gly)10]3. Protein Science 11:262-270. http://doi.org/10.1110/ps.32602

Boonstra, S., P. R. Onck \& E. van der Giessen (2016) CHARMM TIP3P Water Model Suppresses Peptide Folding by Solvating the Unfolded State. The Journal of Physical Chemistry B 120:3692-3698. http://doi.org/10.1021/acs.jpcb.6b01316

Chen, W., H. Yu, S. Y. Lee, T. Wei, J. Li \& Z. Fan (2018) Nanocellulose: a promising nanomaterial for advanced electrochemical energy storage. Chem Soc Rev 47:2837-2872. http://doi.org/10.1039/C7CS00790F

Cudjoe, E., M. Younesi, E. Cudjoe, O. Akkus \& S. J. Rowan (2017) Synthesis and Fabrication of Nanocomposite Fibers of Collagen-Cellulose Nanocrystals by Coelectrocompaction. Biomacromolecules 18:1259-1267. http://doi.org/10.1021/acs.biomac.7b00005

DeLoid, G. M., X. Cao, R. M. Molina, D. I. Silva, K. Bhattacharya, K. W. Ng, S. C. J. Loo, J. D. Brain \& P. Demokritou (2019) Toxicological effects of ingested nanocellulose in in vitro intestinal epithelium and in vivo rat models. Environ Sci Nano 6:2105-2115. http://doi.org/10.1039/c9en00184k

Dorishetty, P., R. Balu, S. S. Athukoralalage, T. L. Greaves, J. Mata, L. de Campo, N. Saha, A. C. W. Zannettino, N. K. Dutta \& N. R. Choudhury (2020) Tunable Biomimetic Hydrogels from Silk Fibroin and Nanocellulose. ACS Sustainable Chemistry \& Engineering 8:2375-2389. http://doi.org/10.1021/acssuschemeng.9b05317

El-Sayed, R., K. Bhattacharya, Z. Gu, Z. Yang, J. K. Weber, H. Li, K. Leifer, Y. Zhao, M. S. Toprak, R. Zhou \& B. Fadeel (2016) Single-Walled Carbon Nanotubes Inhibit the Cytochrome P450 Enzyme, CYP3A4. Sci Rep 6:21316. http://doi.org/10.1038/srep21316

Feng, M., D. R. Bell, J. Luo \& R. Zhou (2017) Impact of graphyne on structural and dynamical properties of calmodulin. Phys Chem Chem Phys 19:10187-10195. http://doi.org/10.1039/c7cp00720e

Fernandes, S. Q. \& C. M. R. Madhuranthakam (2020) Molecular Dynamics Simulation of a Superhydrophobic Cellulose Derivative Targeted for Eco-Friendly Packaging Material. Macromolecular Theory and Simulations 30:2000056. http://doi.org/10.1002/mats.202000056

Ge, L., Y. Xu, X. Li, L. Yuan, H. Tan, D. Li \& C. Mu (2018) Fabrication of Antibacterial Collagen-Based Composite Wound Dressing. ACS Sustainable Chemistry \& Engineering 6:9153-9166. http://doi.org/10.1021/acssuschemeng.8b01482

Golmohammadi, H., E. Morales-Narváez, T. Naghdi \& A. Merkoçi (2017) Nanocellulose in Sensing and Biosensing. Chemistry of Materials 29:5426-5446. http://doi.org/10.1021/acs.chemmater.7b01170

Gomes, T. C. F. \& M. S. Skaf (2012) Cellulose-Builder: A toolkit for building crystalline structures of cellulose. Journal of Computational Chemistry 33:1338-1346. http://doi.org/10.1002/jcc.22959

Gu, Z., P. De Luna, Z. Yang \& R. Zhou (2017) Structural influence of proteins upon adsorption to MoS2 nanomaterials: comparison of MoS2 force field parameters. Phys Chem Chem Phys 19:3039-3045. 
http://doi.org/10.1039/c6cp05260f

Gu, Z., W. Li, L. Hong \& R. Zhou (2016) Exploring biological effects of MoS2 nanosheets on native structures of alpha-helical peptides. J Chem Phys 144:175103. http://doi.org/10.1063/1.4948459

Gu, Z., W. Song, S. H. Chen, B. Li, W. Li \& R. Zhou (2019) Defect-assisted protein HP35 denaturation on graphene. Nanoscale 11:19362-19369. http://doi.org/10.1039/c9nr01143a

He, Z., J. Li, S. H. Chen \& R. Zhou (2019) Surface Inhomogeneity of Graphene Oxide Influences Dissociation of Abeta16-21 Peptide Assembly. J Phys Chem B 123:9098-9103. http://doi.org/10.1021/acs.jpcb.9b07359

Hess, B., H. Bekker, H. Berendsen \& J. Fraaije (2008) LINCS: A linear constraint solver for molecular simulations - Hess - 1998 - Journal of Computational Chemistry - Wiley Online Library. Journal of Chemical Theory \& Computation 4:1463-1472.

Hooft, R., C. Sander \& G. Vriend (1997) Objectively judging the quality of a protein structure from a Ramachandran plot. Computer Applications in the Biosciences Cabios 13:425-30.

Hub, J. S. (2015) g_wham-A Free Weighted Histogram Analysis Implementation Including Robust Error and Autocorrelation Estimates. Journal of Chemical Physics 6:3713-3720.

Humphrey, W., A. Dalke \& K. Schulten (1996) VMD: Visual molecular dynamics. j mol graph 14:33-38.

Jorgensen, W. L., J. Chandrasekhar, J. D. Madura, R. W. Impey \& M. L. Klein (1983) Comparison of simple potential functions for simulating liquid water. The Journal of Chemical Physics 79:926-935. http://doi.org/10.1063/1.445869

Köppen, S., B. Ohler \& W. Langel (2007) Adsorption of Collagen Fragments on Titanium Oxide Surfaces: A Molecular Dynamics Study. Zeitschrift für Physikalische Chemie 221:3-20. http://doi.org/10.1524/zpch.2007.221.1.3

Lee, A., A. R. Hudson, D. J. Shiwarski, J. W. Tashman \& A. W. Feinberg (2019) 3D bioprinting of collagen to rebuild components of the human heart. Science 365:482-487.

Lee, S., A. Tran, M. Allsopp, J. B. Lim, J. Hénin \& J. B. Klauda (2014) CHARMM36 United Atom Chain Model for Lipids and Surfactants. The Journal of Physical Chemistry $B$ 118:547-556. http://doi.org/10.1021/jp410344g

Li, H., W. Cheng, K. Liu, L. Chen, Y. Huang, X. Wang, Z. Lv, J. He \& C. Li (2017) Reinforced collagen with oxidized microcrystalline cellulose shows improved hemostatic effects. Carbohydr Polym 165:30-38. http://doi.org/10.1016/j.carbpol.2017.02.023

Lin, Y. K. \& D. C. Liu (2006) Comparison of physical-chemical properties of type I collagen from different species. Food Chemistry 99:244-251. http://doi.org/10.1016/j.foodchem.2005.06.053

Liu, C.-Y., D. Goto, C. Hongo, T. Matsumoto \& T. Nishino (2018) Collagen/Cellulose Nanofiber Blend Scaffolds Prepared at Various $\mathrm{pH}$ Conditions. ACS Applied Bio Materials 1:1362-1368. http://doi.org/10.1021/acsabm.8b00302

Liu, D., X. Dong, B. Han, H. Huang \& M. Qi (2020a) Cellulose nanocrystal/collagen hydrogels reinforced by anisotropic structure: Shear viscoelasticity and related strengthening mechanism. Composites Communications 21:100374. http://doi.org/10.1016/j.coco.2020.100374

Liu, D., X. Dong, H. Liu, Y. Zhao \& M. Qi (2020b) Effect of pore orientation on shear viscoelasticity of cellulose nanocrystal/collagen hydrogels. Journal of Applied Polymer Science 138:49856. http://doi.org/10.1002/app.49856

Luan, B., T. Huynh \& R. Zhou (2016) Potential Interference of Protein-Protein Interactions by Graphyne. J Phys Chem B 120:2124-31. http://doi.org/10.1021/acs.jpcb.5b11449

Malaspina, D. C. \& J. Faraudo (2019) Molecular insight into the wetting behavior and amphiphilic character of cellulose nanocrystals. Advances in Colloid and Interface Science 267:15-25. 
http://doi.org/10.1016/j.cis.2019.02.003

Manhas, N., K. Balasubramanian, P. Prajith, P. Rule \& S. Nimje (2015) PCL/PVA nanoencapsulated reinforcing fillers of steam exploded/autoclaved cellulose nanofibrils for tissue engineering applications. RSC Advances 5:23999-24008. http://doi.org/10.1039/c4ra17191h

Mark, P. \& L. Nilsson (2001) Structure and Dynamics of the TIP3P, SPC, and SPC/E Water Models at 298 K. The Journal of Physical Chemistry A 105:9954-9960.

Mathesh, M., B. Luan, T. O. Akanbi, J. K. Weber, J. Liu, C. J. Barrow, R. Zhou \& W. Yang (2016) Opening Lids: Modulation of Lipase Immobilization by Graphene Oxides. ACS Catalysis 6:4760-4768. http://doi.org/10.1021/acscatal.6b00942

Nosé, S. \& M. L. Klein (2006) Constant pressure molecular dynamics for molecular systems. Molecular Physics 50:1055-1076. http://doi.org/10.1080/00268978300102851

Otuechere, C. A., A. Adewuyi, O. L. Adebayo \& I. A. Ebigwei (2020) In vivo hepatotoxicity of chemically modified nanocellulose in rats. Hum Exp Toxicol 39:212-223. http://doi.org/10.1177/0960327119881672

Parrinello, M. \& A. Rahman (1981) Polymorphic transitions in single crystals: A new molecular dynamics method. Journal of Applied Physics 52:7182-7190. http://doi.org/10.1063/1.328693

Petersen, H. G. (1995) Accuracy and efficiency of the particle mesh Ewald method. The Journal of Chemical Physics 103:3668-3679. http://doi.org/10.1063/1.470043

Roux, B. (1995) The calculation of the potential of mean force using computer simulations. Computer Physics Communications 91:275-282.

Sai, T. \& K. Fujita (2020) A review of pulmonary toxicity studies of nanocellulose. Inhal Toxicol 32:231-239. http://doi.org/10.1080/08958378.2020.1770901

Salimi, S., R. Sotudeh-Gharebagh, R. Zarghami, S. Y. Chan \& K. H. Yuen (2019) Production of Nanocellulose and Its Applications in Drug Delivery: A Critical Review. ACS Sustainable Chemistry \& Engineering 7:15800-15827. http://doi.org/10.1021/acssuschemeng.9b02744

Sorushanova, A., L. M. Delgado, Z. Wu, N. Shologu, A. Kshirsagar, R. Raghunath, A. M. Mullen, Y. Bayon, A. Pandit, M. Raghunath \& D. I. Zeugolis (2019) The Collagen Suprafamily: From Biosynthesis to Advanced Biomaterial Development. Adv Mater 31:e1801651. http://doi.org/10.1002/adma.201801651

Tang, J., Y. Song, F. Zhao, S. Spinney, J. da Silva Bernardes \& K. C. Tam (2019) Compressible cellulose nanofibril (CNF) based aerogels produced via a bio-inspired strategy for heavy metal ion and dye removal. Carbohydr Polym 208:404-412. http://doi.org/10.1016/j.carbpol.2018.12.079

Van Der Spoel, D., E. Lindahl, B. Hess, G. Groenhof, A. E. Mark \& H. J. Berendsen (2005) GROMACS: fast, flexible, and free. J Comput Chem 26:1701-18. http://doi.org/10.1002/jcc.20291

Vermaas, J. V., M. F. Crowley \& G. T. Beckham (2019) A Quantitative Molecular Atlas for Interactions Between Lignin and Cellulose. ACS Sustainable Chemistry \& Engineering 7:19570-19583. http://doi.org/10.1021/acssuschemeng.9b04648

Zhang, W., X. C. Wang, X. Y. Li, L. L. Zhang \& F. Jiang (2020) A 3D porous microsphere with multistage structure and component based on bacterial cellulose and collagen for bone tissue engineering. Carbohydr Polym 236:116043. http://doi.org/10.1016/j.carbpol.2020.116043

Zhang, Y., H. He, Y. Liu, Y. Wang, F. Huo, M. Fan, H. Adidharma, X. Li \& S. Zhang (2019) Recent progress in theoretical and computational studies on the utilization of lignocellulosic materials. Green Chemistry 21:9-35. http://doi.org/10.1039/c8gc02059k 
Table of Content

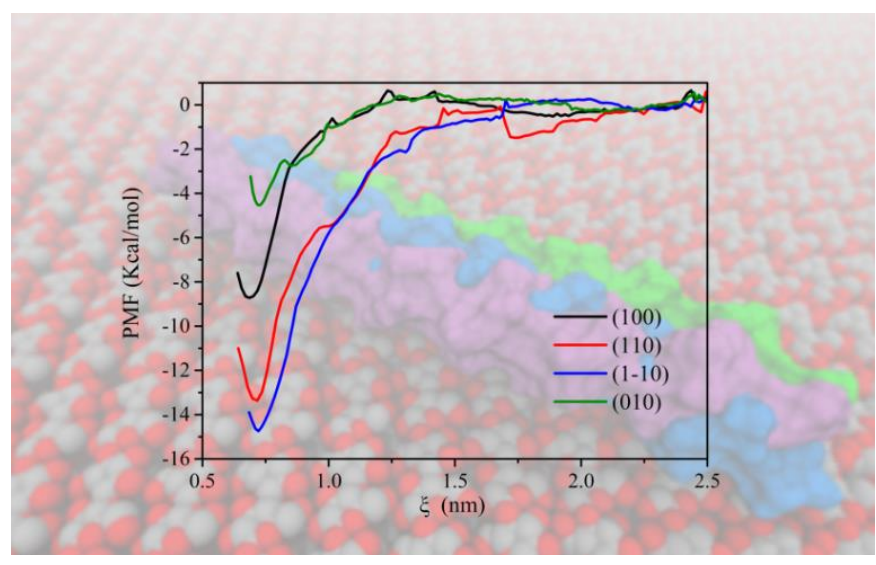

